\title{
Immune evasion through competitive inhibition: the shielding effect of cancer non-stem cells
}

\author{
Irina Kareva \\ Newman Lakka Institute, Floating Hospital for Children at Tufts Medical Center, \\ Boston, MA 02111
}

\begin{abstract}
It has been recently proposed that the two emerging hallmarks of cancer, namely altered glucose metabolism and immune evasion, may in fact be fundamentally linked. This connection comes from up-regulation of glycolysis by tumor cells, which can lead to active competition for resources in the tumor microenvironment between tumor and immune cells. Here it is further proposed that cancer stem cells (CSCs) can circumvent the anti-tumor immune response by creating a "protective shield" of non-stem cancer cells around them. This shield can protect the CSCs both by creating a physical barrier between them and cytotoxic lymphocytes (CTLs), and by promoting competition for the common resources, such as glucose, between non-stem cancer cells and CTLs. The implications of this hypothesis are investigated using an agent-based model, leading to a prediction that relative CSC to non-CSC ratio will vary depending on the strength of the host immune response. A discussion of possible therapeutic approaches concludes the paper, suggesting that a chemotherapeutic regimen consisting of regular pulsed doses, i.e., metronomic chemotherapy, would yield the best clinical outcome by removing the "protective shield" and thus allowing CTLs to most effectively reach and eliminate CSCs.
\end{abstract}

Keywords: cancer immunoediting, cancer stem cells, agent-based model

\section{Introduction}

Cancer stem cells (CSCs) is a small subset of self-sustaining cells within the tumor with unlimited replicative capacity that have been implicated in driving tumor progression. These cells can both self-renew, maintaining the 
stem cell pool within the tumor, and differentiate into non-tumorogenic heterogeneous cancer cell types that constitute most of cancer cells in the tumor (Yamashita et al. (2007)). Moreover, different tumors have been observed to have varying proportions of CSCs, depending on the tissue of origin, ranging from 1\% (Cho et al. (2008)) to 25\% (Quintana et al. (2008)), with the proportions increasing with tumor grade (Thon et al. (2010), Harris et al. (2008)). One possible explanation for this observation lies in the proposition that stemness is a state as opposed to a cell type (Zipori (2004)), and stem cells can change states and dediffernetiate under different conditions, such as fluctuating tissue oxygenation (Shoshani et al. (2014), Spencer et al. (2014)) and changes in cell density (Ko et al. (2009), Shoshani et al. (2014), Wakao et al. (2011)). Low pH has also been proposed to be sufficient for cell reprogramming; however, the papers have been retracted (Obokata et al. (2014a),Obokata et al. (2014b)) and the claim awaits further investigation.

Another, perhaps complementary explanation for varying proportions of CSCs within different tumors, concerns with the effects of the environment, in which CSCs find themselves in the tumor. The components of the environment, in addition to oxygen availability, include also access to nutrients, spatial limitations, and interactions with the immune system, which will be the subject of the model that is proposed here.

There are a number of mechanisms that have been implicated in preventing budding tumors from growing, including genetic mechanisms (Almog (2010), Almog et al. (2009)); lack of angiogenesis (Naumov et al. (2006b), Naumov et al. (2006a)), anti-tumor immunity (Dunn et al. (2004), Ruffell et al. (2010)); the latter will be the focus of this paper. However, as the cytotoxic cells of the immune system attempt to eliminate the tumor, they effectively select for non-immunogenic clones, thus delaying but not arresting tumor progression, a process that has become known as tumor immunoediting (Dunn et al. (2004), Kim et al. (2007), Schreiber et al. (2011)). Moreover, tumors that have been "edited" in this fashion are typically more aggressive than their "unedited" counterparts due to selection for more immunoresistant clones, a property first demonstrated by Shankaran and colleagues (Shankaran et al. (2001)).

It has been recently proposed that the process of immunoediting may additionally be driven by competition for resources between cancer and immune cells in the tumor microenvironment (Kareva (2014)). Specifically, the following microenvironmentally-based immunoediting scenario has been suggested: 
1) As the tumor increases in size while not yet recognized by the cytotoxic cells of the immune system, it begins to outgrow its blood supply, causing the formation of oxygen-deprived regions in the tumor interior.

2) Intra-tumoral hypoxia causes the affected tumor cells to turn to glycolysis as a primary mode of glucose metabolism; an event known to be accompanied by an up to 30-fold up-regulation of the activity of glucose transporters (Ganapathy et al. (2009)).

3) Once activated, the CTLs first attack the topologically accessible outer rim of the tumor, what we term the "protective shield", thus effectively exposing the tumor's glycolytic core.

4) The exposed glycolytic tumor cells with up-regulated glucose transporters now actively compete with CTLs for glucose.

5) In a nutrient-deprived state, CTLs are less able to perform their tumoricidal function, allowing the tumor as a whole to evade immune attack.

In this way, the process of immune evasion may be the result of a competition for resources in the tumor microenvironment. Possible dynamical regimes and further implications of this hypothesis have been investigated in (Kareva (2014)).

Here, this construct is expanded to incorporate cancer stem cells (CSCs). Because CSCs comprise a relatively small fraction of the tumor population on account of their high probability of asymmetric division, producing one CSC and one non-stem cell (Morrison and Kimble (2006), Yamashita et al. (2007)), they will tend to surround themselves with non-stem progeny, which then could follow the aforementioned immunoediting scenario. The nonCSCs will then protect CSCs from cytotoxic lymphocytes both by creating a physical barrier between CSCs and CTLs, and by out-competing CTLs for essential nutrients. These considerations are summarized in Figure 1.

The proposed theoretical framework allows making predictions about CSC content in different tumors with regards to the immune response, which can be of vital importance, as CSCs, the driving cells in cancer progression, are believed to be more resistant to cytotoxic therapy than non-CSCs (O Hare et al. (2006), Oravecz-Wilson et al. (2009),Li et al. (2008), Diehn et al. (2009)). In this case, if CSCs produce too few non-CSCs, protection from the immune system could be insufficient. If CSCs produced too many non-CSCs, then the progeny may themselves become limiting to CSC expansion, out-competing CSCs for space and nutrients, and thus offsetting their benefit as an immune shield. Successful tumors might therefore be predicted to be those that have some optimal intermediate CSC-to-progeny proportion. 
In addition, this proportion may well be different for different initial states of the immune system.

To test these ideas, an agent-based model is implemented, incorporating the foregoing and other basic assumptions about the properties of CSCs, non-CSCs, immune cells and their interactions with each other and with the resources in the tumor microenvironment. We use this model to illustrate the proposed immunoediting construct and in particular, to evaluate the proposition that varying proportions of CSCs to non-CSCs might be expected in successful tumors under different immune conditions.

\section{Model Description}

Here we build upon a previously introduced agent-based model (Aktipis et al. (2012)), adapting it to describe the interactions between cancer stem cells (CSCs), aerobic and glycolytic non-stem cancer cells (non-CSCs), and cytotoxic lymphocytes (CTLs) with each other and their microenvironment. The model is implemented in Netlogo 5.0.2, a freely available agent-based modeling platform (Wilensky and Rand (2009)). Space in this simulation is represented by a 2-dimensional 51x51 lattice. Each patch (square) in the lattice represents a discrete microenvironment, the properties of which are detailed below, for a total of 2601 microenvironments. Cells occupy coordinates in continuous space, and more than one cell can occupy a single microenvironment. Time is modeled in discrete steps.

\subsection{Parameter estimation}

Parameter estimation is a common challenge in analyzing results from computational models. One particular difficulty lies in the fact that the estimates obtained in an experimental setting are typically a result of a multitude of complex interconnected processes, which cannot always be separated out for the purposes of a conceptual proof-of-concept model. We address this issue in the following ways. A number of parameters, such as cell growth rates, were previously estimated in a model introduced and analyzed in (Aktipis et al. (2012)), where the authors utilize an agent-based model to evaluate the impacts of disregulated glucose metabolism on overall tumor dynamics. We modify their proposed model, shifting the focus of our investigation away

from evolution of cell motility and instead introducing immune cells and 
cancer stem cells as active competitors for glucose in the tumor microenvironment. Nevertheless, parameters that pertain to tumor growth rates and glucose consumption rates can be well translated into our model.

For a number of other parameters, while it may not always be possible to obtain exact values for reasons outlined above, we attempt to make estimations based on the parameters relative values to each other. That is, while we may not be able to estimate exact rates of glucose consumption by cytotoxic immune cells, for instance, we can nevertheless support the notion that highly proliferative CTLs, just as glycolytic tumor cells, consume more energy than do aerobic tumor cells (Fox et al. (2005)). Morover, Buttgereit and Brand (Buttgereit and Brand (1995)) reported that in a model of concanavlin A-stimulated thymocytes, where it is possible to account for over $80 \%$ of ATP consumption, protein synthesis was the most sensitive to limitations in glucose supply, followed by DNA and RNA synthesis and then by ion transport across plasma membrane. Motility also has been reported to incur significant ATP costs for immune cells due to energy requirements for cytoskeleton rearrangement during movement (Buttgereit et al. (2000)) Therefore, while we may not be able to obtain the exact values for the costs of motility and cytotoxicity for CTLs, we can nevertheless assert that these costs exist, and are incorporated as such. Moreover, since we do not have exact estimates for these costs, they are taken to be a unit of energy each, an assumption which can be relaxed or modified pending data availability.

The specific assumptions for each of the cell types and for the microenvironments are as follows:

Properties of aerobic non-CSCs: Aerobic non-CSCs require glucose and oxygen for survival and glucose for proliferation, although they have lower glucose requirements than glycolytic non-CSCs. They die at some natural rate, as well as after a certain number of divisions, and can also be killed by CTLs. They can 'switch to the glycolytic mode of metabolism under conditions of oxygen deprivation. When a cell divides, the daughter cell moves to a random neighboring patch.

Properties of glycolytic non-CSCs: Glycolytic non-CSCs do not require oxygen, but require glucose for survival and proliferation; they consume more glucose to support these processes than aerobic non-CSCs. They die at some natural rate, as well as after a certain number of divisions, and can also be killed by CTLs. They can 'switch to the aerobic mode of glucose metabolism under conditions of sufficient oxygen availability. When a cell divides, the daughter cell moves to a random neighboring patch. 
Properties of CSCs: Depending on oxygen availability, CSCs consume oxygen and glucose like aerobic or glycolytic cells. Each CSC division occurs symmetrically with a certain probability. If a CSC divides asymmetrically, the daughter non-CSC cell is initialized to be aerobic, a state which may change. Each cell can die due to nutrient deprivation or be killed by CTLs. CSCs can divide an unlimited number of times. When a CSC divides, the daughter cell moves to a random neighboring patch.

Properties of cytotoxic immune cells: It is assumed that all cytotoxic CTLs rely on glycolysis for glucose metabolism, consuming nutrients at the same rate as glycolytic tumor cells. CTLs can proliferate only under conditions of sufficient glucose supply. They can die from nutrient deprivation. Unlike tumor cells, CTLs are motile and move randomly throughout the microenvironment. As motility is costly, each step to a random neighboring patch costs CTLs a unit of energy. CTLs can kill tumor cells once they encounter them; however, killing a tumor cell also costs a unit of energy. CTLs are replenished at some constant rate when the number of immune cells in the tumor microenvironment becomes too low.

Properties of the microenvironment: On each patch there is a certain amount of glucose and oxygen. Both glucose and oxygen are replenished differentially, depending on the number of cells on the patch. Too many cells on the patch lowers the rate of replenishment, which simulates the crowding effect. Glucose and oxygen are taken up by different cell types at different rates, depending on whether the cell type is aerobic, glycolytic, stem or immune.

It is worth noting that while alternative energy sources, such as glutamine, are available to tumor cells, it has been shown by MacIver and colleagues (MacIver et al. (2008)) that activated T cells are unable to perform their function in the absence of glucose, even in the presence of amply available glutamine. Therefore, glutamine was not incorporated as an energy source in the proposed model as it is not the driving force for competition between tumor and immune cells.

The model is initialized with a single CSC and a pre-set initial number of cytotoxic immune cells placed randomly throughout the lattice. At each time step, the following sequence of steps is realized:

1) Each tumor cell evaluates oxygen availability in its microenvironment, which determines the cell's subsequent mode of glucose metabolism.

2) Each cell consumes glucose, subtracting differentially from the glucose available on the patch and adding a number of energy units to the cell's 
storage, according to its 'metabolic phenotype', i.e., aerobic or glycolytic; glycolytic cells consume glucose, while aerobic cells consume both glucose and oxygen.

3) Cytotoxic lymphocytes move randomly throughout the microenvironment, killing the tumor cells that they encounter; both motility and the process of cell killing have a cost of a unit of energy each; CTLs die if their energy stores are depleted and cannot be replenished.

4) Each cell reproduces with a certain fixed probability, given adequate resources in the microenvironment; the daughter cell moves to a random neighboring patch; if the cell is a CSC, it divides symmetrically or asymmetrically with a certain probability; in case of asymmetric division, the daughter cell is initialized as aerobic non-CSC.

5) Glucose and oxygen are replenished in the microenvironment depending on the number of cells on each patch, with a higher rate of replenishment in the areas of lower cell density.

6) CTLs are replenished by the value of parameter $i_{0}$ if the number of immune cells on the lattice falls below a critical threshold.

Since the purpose of the proposed model is to offer a 'proof-of-concept' as to whether tumor composition can indeed be affected by the initial state of the immune system of the host, parameter values were chosen as relative to each other rather than matching them to fit a particular data set.

It is hypothesized that in successfully formed tumors, the relative proportion of non-CSCs will increase proportionally to the strength of the immune response because CSCs will require the shielding effect of non-CSCs to protect them from CTLs. Specifically, an increase in the proportion of glycolytic non-CSCs is predicted, since they are capable of competing most effectively with CTLs for glucose in the tumor microenvironment.

\section{Results}

The numerical experiments were designed in such a way as to enable evaluation of tumor composition at each time point with respect to the state of the immune system. The initial state of the immune response was modeled with parameter $i_{0}=0,10,20,30,35,40$. For each $i_{0}, 20$ simulations were run for 1000 time steps, and the mean number of CSCs, aerobic and glycolytic non-CSCs were reported; only the data for tumors that had at least one CSC at $t=1000$ were collected. The data is represented in two types of plots: the 
change over time of tumor composition for each respective $i_{0}$ and the final ratio of CSC to non-CSC at $t=1000$ for each $i_{0}$.

\subsection{Proportion of glycolytic cells increases with the strength of the immune response}

In Figure 2, it can be observed that over time, the proportion of glycolytic non-CSCs becomes increasingly larger for higher $i_{0}$. However, the largest ratio of CSC:non-CSC at $t=1000$ occurs at $i_{0}=25$, which is the intermediate value of simulated strength of immune response (Figure 3 ).

The following explanation for this phenomenon is proposed:

At low values of $i_{0}$, aerobic non-CSCs that are located on the tumor rim have the greatest competitive advantage compared to other tumor cells, as they have the most access to oxygen and glucose compared to both CSCs and glycolytic non-CSCs inside the tumor. Therefore, since they are not 'sacrificed to the immune system, aerobic non-CSCs outcompete CSCs.

At high values of $i_{0}$, the tumor is not able to grow to a large enough size to create sufficiently large hypoxic regions that would allow for the appearance of large clusters of glycolytic non-CSCs that would present sufficient competition to cytotoxic lymphocytes. In this case, the non-CSC 'shield would not be strong enough to contain CTLs.

At "intermediate" values of $i_{0}$, the tumor can grow to be large enough in size to have sufficiently large hypoxic regions to require up-regulation of purely glycolytic mode of glucose metabolism in a sufficiently large number of non-CSCs. At the same time, the immune system removes enough of the aerobic non-CSCs at the outer rim of the tumor to prevent them from outcompeting the rest of the tumor cells. Therefore, one should expect the largest proportions of CSC in hosts with "intermediate" states of immune system. (Noticeably, these observations can be made based on assumptions of relative values of parameters, without requiring exact parameter estimation from specific data sets).

\subsection{Variability in sensitivity to oxygen does not affect final tumor composi- tion regardless of the state of the immune system}

In the previous set of in silico experiments, it was assumed that all cells have a fixed threshold of sensitivity to oxygen and therefore revert to either aerobic or glycolytic mode of glucose metabolism based on a criterion that is uniform for all cells. It is however possible that if there were heterogeneity among cells based on this metric, one could observe selection for cells that 
are either more or less sensitive to oxygen concentrations in their microenvironment depending on the state of the immune response. That is, one could observe selection towards lower oxygen sensitivity thresholds (described by the value of parameter $\omega$ ) under the conditions of increased selective pressure from the immune system.

In order to evaluate this hypothesis, the above-described protocol was modified to introduce mutations in parameter every time a tumor cell divides. The changes in the mean value of $\omega$ were recorded for all three cell types for all $i_{0}$. The overall tumor composition changed in the same way with respect to different $i_{0}$ as it did for the case of constant $\omega$ (Figure 4), and the largest proportion of CSCs was also observed for the intermediate values of $i_{0}$ (Figure 5). The mean value $\omega$ for glycolytic cancer cells did oscillate increasingly with stronger immune response (Figure 6). However, a 2-tailed type 1 t-test did not reveal any statistically significant changes either in overall tumor composition or in the values of at $t=1000$ for either of the three tumor cell types (Figure 7). These results suggest that it is not oxygen availability but competition for glucose, both within the tumor and between cancer and immune cells, that is the driving force behind the observed dynamics of immune evasion.

\section{Discussion}

Here a theoretical framework is proposed, where CSCs can escape recognition by cytotoxic lymphocytes by creating a 'shield of differentiated nonCSCs around them. These shielding cells not only provide a physical barrier to the immune cells but also create a local nutrient flux, thereby starving CTLs out of functionality. Specifically, we proposed that if a CSC divided asymmetrically before it was recognized by the CTLs, and if the progeny grew to form a sufficiently large tumor to cause the appearance of hypoxic regions and consequent reversion by many of the tumor cells to purely glycolytic mode of glucose metabolism, then, once the immune cells eventually clear the aerobic cells on the outer rim of the tumor, they will encounter

competition for glucose from glycolytic cancer cells. Successful acquisition of the shared glucose, made possible by up-regulation of nutrient transporters of glycolytic cancer cells while in the hypoxic regions of the tumor, could cause incapacitation of CTLs due to starvation, thereby allowing for continued unrestrained tumor growth (see Figure 1). 
In order to evaluate the proposed hypothesis, an agent-based model was created, focusing on interactions between CSCs, aerobic and glycolytic nonCSCs with varying glucose demands, and motile cytotoxic immune cells, whose functionality is nutrient dependent. Simulations were run for 1000 time steps, for various initial states of the immune system of the host, ranging from complete immunodeficiency to extremely strong immune response. Tumor composition was then evaluated for all of the initial immune states.

The proportion of glycolytic non-CSCs increased dramatically with increased immune response (Figure 2), supporting the initial hypothesis. Moreover, the proportion of CSCs was largest not for the strongest or weakest states of the immune response but for intermediate ones (Figure 3), supposedly because in this case the tumor can grow large enough to create hypoxic regions but is still sufficiently small to prevent the non-stem progeny from out-competing CSCs.

Next, we hypothesized that if each cell had an individual threshold of sensitivity to oxygen, which would determine oxygen-based threshold for a glycolytic switch, then over time selection towards cells with lower threshold could be observed. However, while the threshold of sensitivity to oxygen $\omega$ did change slightly over time, the changes both in tumor composition, as well as in the mean values of $\omega$ for all three cell types was not statistically significant (Figures 6 and 7), suggesting that oxygen availability is less of a crucial determinant of the outcome of tumor progression compared to glucose.

It is of course not possible to claim that competition for common nutrients is the sole mechanism whereby CSCs can avoid recognition by the immune system. Instead, we propose that competition for resources can contribute to immune evasion. Noticeably, modifying the model to increase or decrease the actual costs of motility and cytotoxicity of CTLs does not affect the predicted pattern of behavior (data not reported). Similarly, modification of the level of possible infiltration of the tumor by CTLs based on crowding effects did not result in qualitative changes in dynamics (data not reported). This can be explained by the fact that even when CTLs are physically able to infiltrate the simulated tumor, they are quickly starved by glycolytic tumor cells in the hypoxic tumor core, thus exhibiting the same qualitative dynamics.

Another important consideration is relative sensitivity of CSCs to CTLs. The literature on this account is inconclusive: while a number of authors report lower sensitivity of CSCs to being recognized by CTLs compared to non-CSCs (Di Tomaso et al. (2010), Chikamatsu et al. (2011)), it is unclear whether this is solely an intrinsic property of CSCs, such as lower expression 
of MHC-II molecules (Chikamatsu et al. (2011)), or a by-product of the 'shielding effect of non-CSCs. Other groups report efficient recognition of CSCs by CTLs (Inoda et al. (2011)), which suggests that this is a question that in fact pends further investigation.

One possible way to understand the interactions of CSCs with their environment is from the point of view of normal processes that occur during embryogenesis or tissue regeneration. Tissue injury is often accompanied by hypoxia due to damage to blood vessels, which results in local acidosis as the cells downstream from the damaged vessels become oxygen deprived. Lower $\mathrm{pH}$ appears to create a locally immunosuppressive environment (Kareva and Hahnfeldt (2013)), providing an opportunity for tissues to rebuild themselves. Normalized vascularization after tissue regeneration would allow for exposure of adult stem cells to immune cells, thus providing an additional regulatory mechanism to stop generation of new tissues. Tumors in turn could harness this mechanism, continuing to grow through creating a hypoxic environment, inadvertently protecting CSCs. This hypothesis will require further investigation.

\subsection{Evolutionary perspective}

It has become increasingly recognized and accepted that tumor dynamics is governed largely by the process of Darwinian selection, where tumor cells experience and respond to various selective pressures, such as competition for resources with each other and somatic cells, interaction with predators (the immune system), migration (metastases), all within the 'ecosystem of the human body (Pienta et al. (2008), Breivik (2001), Greaves and Maley (2012), Nowell (1976), Calabrese et al. (2004), Greaves (2010), Kareva (2011b), Merlo et al. (2006)). One of the well-developed analytical approaches to understanding evolving populations is evolutionary game theory, which has already been partially applied to cancer (Basanta et al. (2008b), Basanta et al. (2008a), Mansury et al. (2006), Vincent and Brown (2005), Gatenby and Vincent (2003), Kareva (2011a)). The main premise of this approach is the assumption that individuals, such as cells, operate for immediate optimization of their payoff, which in the context of evolutionary game theory is fitness, i.e., survival and the number of progeny. Individuals may use different strategies to achieve this goal, and understanding of these strategies and their immediate consequences can provide understanding about why a system is evolving in a particular direction. 
In the context of the proposed model, we assume that it is CSCs that "strive" to maximize their fitness and produce a maximal number of CSCs. This goal can be achieved using two strategies: symmetric or asymmetric division, yielding either two CSCs per cell division, or one CSC and one nonCSC, respectively. Symmetric division provides an immediately higher CSC yield. Asymmetric division may allow for survival of the progeny in the long term.

From this point of view, in the absence of the immune response, or if it did not affect the growth dynamics CSCs, one would expect eventual CSC enrichment, as is predicted by a number of previously published theoretical models (Enderling et al. (2012), Hillen et al. (2013)), since it is symmetric division that would allow CSCs to maximize their fitness. However, in the presence of the immune response, the "optimal strategy" becomes mixed, since in order to maximize their fitness CSCs have to divide asymmetrically to protect themselves from the immune cells. Thus, the optimal "equilibrium strategy", i.e., the number of times the CSC would need to divide symmetrically or asymmetrically in order to maximize its fitness, would be different depending on the initial state of the immune response. Perhaps, manipulating the tumor microenvironment in such a way as to select for the increased asymmetric CSC division could be a potentially fruitful strategy, making tumors more vulnerable to anti-cancer therapies.

\subsection{Therapeutic implications}

Cancer stem cells are believed to be the driving force behind unrestrained tumor growth, as they are also resistant to a number of cytotoxic therapies (Li et al. (2008)Moncharmont et al. (2012), McCord et al. (2009), Marcato et al. (2009), Rich (2007)). One possible way to circumvent this problem could involve taking advantage of the immune cells, which might be able to eliminate CSCs, even if less efficiently compared to non-CSCs (Inoda et al. (2011), Schatton and Frank (2009), Hirohashi et al. (2010), Todaro et al. (2009)). However, if the proposed construct is correct, CTLs are unable to do so because of the 'shield' of differentiated tumor cells that restricts their access to CSCs. Therefore, the optimal therapeutic strategy in this case would be not the one to cause the most extensive cell mortality but the one that will facilitate the access of cytotoxic immune cells to CSCs.

Two main considerations about CSCs and CTLs need to be taken into account. Firstly experimental evidence suggests that CSCs are to be primarily found in the hypoxic areas of the tumor (Heddleston et al. (2009), Lin 
and Yun (2010)), where they are the most 'protected from the immune cells. Secondly, extensive cytotoxic therapies are highly damaging to the immune cells themselves (Doloff and Waxman (2012), Chen et al. (2014)), and consequently, gaining access the tumor core and the CSCs would be ineffective if there remain no immune cells that could then eliminate the CSCs.

The three major approaches that can be taken in chemotherapy administration are MTD (maximum tolerated dose), metronomic, and continuous therapy, which is a particular case of the metronomic therapy.

As the name suggests, MTD involves administering the highest possible dose of cytotoxic drugs that a patient can tolerate, with the intention of damaging a maximal number of cells and expectation that the recovery of normal tissues will occur faster than that of the tumor cells. The major drawback of MTD from the point of view of the considerations, outlined above, is the damage that it causes to the immune cells (Doloff and Waxman (2012), Chen et al. (2014)). Therefore, even if this were a good way to reach the CSCs, it is very likely that the time it would take for the anti-tumor immune cells to recover would be greater than the time it would take for the CSCs to form another "protective shield". Noticeably, these predictions should hold as long as CSCs can be recognized by CTLs.

Continuous therapy involves administering very low doses of chemotherapy on a daily basis, ensuring constant presence of the cytotoxic drug in the patient's body. While this approach is much less toxic to the patient, it still has a problem of allowing no time for the recovery of anti-tumor immune cells.

Metronomic therapy provides an intermediate case between MTD and continuous therapy, and involves administering lower doses of chemotherapy at more frequent intervals, but not on a daily basis. This approach could ensure continual "peeling off" of the outer layers of the tumor, which protect the CSCs, thus giving anti-tumor immune cells access to the tumor core, while allowing sufficient time for CTLs to recover from the damaging effects of the therapy and attack the CSCs, thereby giving the best chance for more successful long-term tumor elimination. Experimental evidence supports these predictions. Specifically, Folkins et al.( Folkins et al. (2007)) demonstrated that out of several treatment regimens administered to athymic nude mice bearing s.c. C6 rat glioma xenografts, only metronomic chemotherapy in combination with direct anti-angiogenic reduced the CSC population; neither targeted anti-angiogenic therapy nor cytotoxic chemotherapy alone reduced the fraction of tumor sphere-forming cells, which correspond to CSCs. 
Furthermore, Vives and colleagues (Vives et al. (2013)) found that an MTD regimen followed by metronomic chemotherapy (a chemo-switch schedule), was more effective in blocking metastatic dissemination in an orthotopic pancreatic adenocarcinoma model compared to MTD treatment. An increase in TSP-1 expression and a decrease in the number of CSCs bearing CD133+ and CD133+/CD44+/CD24+ markers was also seen, indicating that a cytoreductive MTD regimen followed by metronomic chemotherapy may be a promising strategy for eradicating chemotherapy-resistant cancer stem cells.

Intriguingly, despite rapidly accumulating evidence of superiority of metronomic chemotherapy regimen over MTD, there is still much resistance to the concept among clinicians, a concern that has been raised at several recent workshops, devoted to this topic (Hahnfeldt et al. (2013), Pasquier et al. (2011)). It is likely that such resistance comes largely in part from lack of understanding of theoretical underpinnings of metronomic chemotherapy. Mechanistic explanations, such as the one provided here, might assist in increasing acceptance of metronomic regimen as a primary therapeutic modality. 


\section{Acknowledgements}

This research was partially supported by the Office of Science (BER), U.S. Department of Energy, under Award Number DE-SC0001434 (to Philip Hahnfeldt, IKs post-doctoral mentor) and Award Number DE-SC0002606 (to Lynn Hlatky), and by a National Cancer Institute grant ICBP 1U54CA149233 (to Lynn Hlatky). The research has been conducted during IKs postdoctoral training at Center of Cancer Systems Biology, St. Elizabeths Medical Center, Boston, MA, 02135, in 2012-2013. The author would also like to thank Philip

Hahnfeldt, Lynn Hlatky, Heiko Enderling and Kathleen Wilkie for extremely helpful suggestions and discussions. 
Aktipis, C. A., Maley, C. C., Pepper, J. W., 2012. Dispersal evolution in neoplasms: the role of disregulated metabolism in the evolution of cell motility. Cancer prevention research 5 (2), 266-275.

Almog, N., 2010. Molecular mechanisms underlying tumor dormancy. Cancer letters 294 (2), 139-146.

Almog, N., Ma, L., Raychowdhury, R., Schwager, C., Erber, R., Short, S., Hlatky, L., Vajkoczy, P., Huber, P. E., Folkman, J., et al., 2009. Transcriptional switch of dormant tumors to fast-growing angiogenic phenotype. Cancer research 69 (3), 836-844.

Basanta, D., Hatzikirou, H., Deutsch, A., 2008a. Studying the emergence of invasiveness in tumours using game theory. The European Physical Journal B-Condensed Matter and Complex Systems 63 (3), 393-397.

Basanta, D., Simon, M., Hatzikirou, H., Deutsch, A., 2008b. Evolutionary game theory elucidates the role of glycolysis in glioma progression and invasion. Cell proliferation 41 (6), 980-987.

Breivik, J., 2001. Don't stop for repairs in a war zone: Darwinian evolution unites genes and environment in cancer development. Proceedings of the National Academy of Sciences 98 (10), 5379-5381.

Buttgereit, F., Brand, M. D., 1995. A hierarchy of atp-consuming processes in mammalian cells. Biochem. J 312, 163-167.

Buttgereit, F., Burmester, G.-R., Brand, M. D., 2000. Bioenergetics of immune functions: fundamental and therapeutic aspects. Immunology today 21 (4), 194-199.

Calabrese, P., Tavaré, S., Shibata, D., 2004. Pretumor progression: clonal evolution of human stem cell populations. The American journal of pathology 164 (4), 1337-1346.

Chen, C.-S., Doloff, J. C., Waxman, D. J., 2014. Intermittent metronomic drug schedule is essential for activating antitumor innate immunity and tumor xenograft regression. Neoplasia 16 (1), 84-IN27.

Chikamatsu, K., Takahashi, G., Sakakura, K., Ferrone, S., Masuyama, K., 2011. Immunoregulatory properties of cd44+ cancer stem-like cells in squamous cell carcinoma of the head and neck. Head \& neck 33 (2), 208-215. 
Cho, R. W., Wang, X., Diehn, M., Shedden, K., Chen, G. Y., Sherlock, G., Gurney, A., Lewicki, J., Clarke, M. F., 2008. Isolation and molecular characterization of cancer stem cells in mmtv-wnt-1 murine breast tumors. Stem cells 26 (2), 364-371.

Di Tomaso, T., Mazzoleni, S., Wang, E., Sovena, G., Clavenna, D., Franzin, A., Mortini, P., Ferrone, S., Doglioni, C., Marincola, F. M., et al., 2010. Immunobiological characterization of cancer stem cells isolated from glioblastoma patients. Clinical Cancer Research 16 (3), 800-813.

Diehn, M., Cho, R. W., Lobo, N. A., Kalisky, T., Dorie, M. J., Kulp, A. N., Qian, D., Lam, J. S., Ailles, L. E., Wong, M., et al., 2009. Association of reactive oxygen species levels and radioresistance in cancer stem cells. nature 458 (7239), 780-783.

Doloff, J. C., Waxman, D. J., 2012. Vegf receptor inhibitors block the ability of metronomically dosed cyclophosphamide to activate innate immunity induced tumor regression. Cancer research 72 (5), 1103-1115.

Dunn, G. P., Old, L. J., Schreiber, R. D., 2004. The three es of cancer immunoediting. Annu. Rev. Immunol. 22, 329-360.

Enderling, H., Hlatky, L., Hahnfeldt, P., 2012. Immunoediting: evidence of the multifaceted role of the immune system in self-metastatic tumor growth. Theor Biol Med Model 9, 31-31.

Folkins, C., Man, S., Xu, P., Shaked, Y., Hicklin, D. J., Kerbel, R. S., 2007. Anticancer therapies combining antiangiogenic and tumor cell cytotoxic effects reduce the tumor stem-like cell fraction in glioma xenograft tumors. Cancer research 67 (8), 3560-3564.

Fox, C. J., Hammerman, P. S., Thompson, C. B., 2005. Fuel feeds function: energy metabolism and the t-cell response. Nature Reviews Immunology 5 (11), 844-852.

Ganapathy, V., Thangaraju, M., Prasad, P. D., 2009. Nutrient transporters in cancer: relevance to warburg hypothesis and beyond. Pharmacology \& therapeutics 121 (1), 29-40.

Gatenby, R. A., Vincent, T. L., 2003. An evolutionary model of carcinogenesis. Cancer Research 63 (19), 6212-6220. 
Greaves, M., 2010. Cancer stem cells: back to darwin? In: Seminars in cancer biology. Vol. 20. Elsevier, pp. 65-70.

Greaves, M., Maley, C. C., 2012. Clonal evolution in cancer. Nature 481 (7381), 306-313.

Hahnfeldt, P., Hlatky, L., Klement, G. L., 2013. Center of cancer systems biology second annual workshoptumor metronomics: timing and dose level dynamics. Cancer research 73 (10), 2949-2954.

Harris, M. A., Yang, H., Low, B. E., Mukherje, J., Guha, A., Bronson, R. T., Shultz, L. D., Israel, M. A., Yun, K., 2008. Cancer stem cells are enriched in the side population cells in a mouse model of glioma. Cancer research 68 (24), 10051-10059.

Heddleston, J. M., Li, Z., McLendon, R. E., Hjelmeland, A. B., Rich, J. N., 2009. The hypoxic microenvironment maintains glioblastoma stem cells and promotes reprogramming towards a cancer stem cell phenotype. Cell Cycle 8 (20), 3274-3284.

Hillen, T., Enderling, H., Hahnfeldt, P., 2013. The tumor growth paradox and immune system-mediated selection for cancer stem cells. Bulletin of mathematical biology 75 (1), 161-184.

Hirohashi, Y., Torigoe, T., Inoda, S., Takahashi, A., Morita, R., Nishizawa, S., Tamura, Y., Suzuki, H., Toyota, M., Sato, N., 2010. Immune response against tumor antigens expressed on human cancer stem-like cells/tumorinitiating cells. Immunotherapy 2 (2), 201-211.

Inoda, S., Hirohashi, Y., Torigoe, T., Morita, R., Takahashi, A., Asanuma, H., Nakatsugawa, M., Nishizawa, S., Tamura, Y., Tsuruma, T., et al., 2011. Cytotoxic t lymphocytes efficiently recognize human colon cancer stem-like cells. The American journal of pathology 178 (4), 1805-1813.

Kareva, I., 2011a. Prisoner's dilemma in cancer metabolism. PloS one 6 (12), e28576.

Kareva, I., 2011b. What can ecology teach us about cancer? Translational oncology 4 (5), 266-270. 
Kareva, I., 2014. Competition driven cancer immunoedition. arXiv preprint q-bio.TO/1407.7853.

Kareva, I., Hahnfeldt, P., 2013. The emerging "hallmarks" of metabolic reprogramming and immune evasion: distinct or linked? Cancer research $73(9), 2737-2742$.

Kim, R., Emi, M., Tanabe, K., 2007. Cancer immunoediting from immune surveillance to immune escape. Immunology 121 (1), 1-14.

Ko, K., Tapia, N., Wu, G., Kim, J. B., Bravo, M. J. A., Sasse, P., Glaser, T., Ruau, D., Han, D. W., Greber, B., et al., 2009. Induction of pluripotency in adult unipotent germline stem cells. Cell Stem Cell 5 (1), 87-96.

Li, X., Lewis, M. T., Huang, J., Gutierrez, C., Osborne, C. K., Wu, M.-F., Hilsenbeck, S. G., Pavlick, A., Zhang, X., Chamness, G. C., et al., 2008. Intrinsic resistance of tumorigenic breast cancer cells to chemotherapy. Journal of the National Cancer Institute 100 (9), 672-679.

Lin, Q., Yun, Z., 2010. Impact of the hypoxic tumor microenvironment on the regulation of cancer stem cell characteristics. Cancer Biol Ther 9 (12), 949-956.

MacIver, N. J., Jacobs, S. R., Wieman, H. L., Wofford, J. A., Coloff, J. L., Rathmell, J. C., 2008. Glucose metabolism in lymphocytes is a regulated process with significant effects on immune cell function and survival. Journal of leukocyte biology 84 (4), 949-957.

Mansury, Y., Diggory, M., Deisboeck, T. S., 2006. Evolutionary game theory in an agent-based brain tumor model: exploring the genotypephenotypelink. Journal of theoretical biology 238 (1), 146-156.

Marcato, P., Dean, C. A., Giacomantonio, C. A., Lee, P. W., 2009. If cancer stem cells are resistant to current therapies, what's next? Future Oncology 5 (6), 747-750.

McCord, A. M., Jamal, M., Williams, E. S., Camphausen, K., Tofilon, P. J., 2009. Cd133+ glioblastoma stem-like cells are radiosensitive with a defective dna damage response compared with established cell lines. Clinical Cancer Research 15 (16), 5145-5153. 
Merlo, L. M., Pepper, J. W., Reid, B. J., Maley, C. C., 2006. Cancer as an evolutionary and ecological process. Nature Reviews Cancer 6 (12), 924935.

Moncharmont, C., Levy, A., Gilormini, M., Bertrand, G., Chargari, C., Alphonse, G., Ardail, D., Rodriguez-Lafrasse, C., Magné, N., 2012. Targeting a cornerstone of radiation resistance: cancer stem cell. Cancer letters 322 (2), 139-147.

Morrison, S. J., Kimble, J., 2006. Asymmetric and symmetric stem-cell divisions in development and cancer. Nature 441 (7097), 1068-1074.

Naumov, G. N., Akslen, L. A., Folkman, J., 2006a. Role of angiogenesis in human tumor dormancy: animal models of the angiogenic switch. Cell cycle 5 (16), 1779-1787.

Naumov, G. N., Bender, E., Zurakowski, D., Kang, S.-Y., Sampson, D., Flynn, E., Watnick, R. S., Straume, O., Akslen, L. A., Folkman, J., et al., 2006b. A model of human tumor dormancy: an angiogenic switch from the nonangiogenic phenotype. Journal of the National Cancer Institute 98 (5), $316-325$.

Nowell, P. C., 1976. The clonal evolution of tumor cell populations. Science 194 (4260), 23-28.

O Hare, T., Corbin, A. S., Druker, B. J., 2006. Targeted cml therapy: controlling drug resistance, seeking cure. Current opinion in genetics \& development 16 (1), 92-99.

Obokata, H., Sasai, Y., Niwa, H., Kadota, M., Andrabi, M., Takata, N., Tokoro, M., Terashita, Y., Yonemura, S., Vacanti, C. A., et al., 2014 a. Retraction: Bidirectional developmental potential in reprogrammed cells with acquired pluripotency. Nature 511 (7507), 112-112.

Obokata, H., Wakayama, T., Sasai, Y., Kojima, K., Vacanti, M. P., Niwa, H., Yamato, M., Vacanti, C. A., 2014b. Retraction: Stimulus-triggered fate conversion of somatic cells into pluripotency. Nature 511 (7507), 112-112.

Oravecz-Wilson, K. I., Philips, S. T., Yilmaz, O. H., Ames, H. M., Li, L., Crawford, B. D., Gauvin, A. M., Lucas, P. C., Sitwala, K., Downing, J. R., et al., 2009. Persistence of leukemia-initiating cells in a conditional knockin 
model of an imatinib-responsive myeloproliferative disorder. Cancer cell $16(2), 137-148$.

Pasquier, E., Kieran, M. W., Sterba, J., Shaked, Y., Baruchel, S., Oberlin, O., Kivivuori, M. S., Peyrl, A., Diawarra, M., Casanova, M., et al., 2011. Moving forward with metronomic chemotherapy: meeting report of the 2nd international workshop on metronomic and anti-angiogenic chemotherapy in paediatric oncology. Translational oncology 4 (4), 203-211.

Pienta, K. J., McGregor, N., Axelrod, R., Axelrod, D. E., 2008. Ecological therapy for cancer: defining tumors using an ecosystem paradigm suggests new opportunities for novel cancer treatments. Translational oncology 1 (4), 158-164.

Quintana, E., Shackleton, M., Sabel, M. S., Fullen, D. R., Johnson, T. M., Morrison, S. J., 2008. Efficient tumour formation by single human melanoma cells. Nature 456 (7222), 593-598.

Rich, J. N., 2007. Cancer stem cells in radiation resistance. Cancer Research 67 (19), 8980-8984.

Ruffell, B., DeNardo, D. G., Affara, N. I., Coussens, L. M., 2010. Lymphocytes in cancer development: polarization towards pro-tumor immunity. Cytokine \& growth factor reviews 21 (1), 3-10.

Schatton, T., Frank, M. H., 2009. Antitumor immunity and cancer stem cells. Annals of the New York Academy of Sciences 1176 (1), 154-169.

Schreiber, R. D., Old, L. J., Smyth, M. J., 2011. Cancer immunoediting: integrating immunity's roles in cancer suppression and promotion. Science 331 (6024), 1565-1570.

Shankaran, V., Ikeda, H., Bruce, A. T., White, J. M., Swanson, P. E., Old, L. J., Schreiber, R. D., 2001. Ifn $\gamma$ and lymphocytes prevent primary tumour development and shape tumour immunogenicity. Nature 410 (6832), $1107-1111$.

Shoshani, O., Ravid, O., Massalha, H., Aharonov, A., Ovadya, Y., PevsnerFischer, M., Leshkowitz, D., Zipori, D., 2014. Cell isolation induces fate changes of bone marrow mesenchymal cells leading to loss or alternatively to acquisition of new differentiation potentials. Stem Cells. 
Spencer, J. A., Ferraro, F., Roussakis, E., Klein, A., Wu, J., Runnels, J. M., Zaher, W., Mortensen, L. J., Alt, C., Turcotte, R., et al., 2014. Direct measurement of local oxygen concentration in the bone marrow of live animals. Nature 508 (7495), 269-273.

Thon, N., Damianoff, K., Hegermann, J., Grau, S., Krebs, B., Schnell, O., Tonn, J.-C., Goldbrunner, R., 2010. Presence of pluripotent cd133; $\sup i+i / \sup i$ cells correlates with malignancy of gliomas. Molecular and Cellular Neuroscience 43 (1), 51-59.

Todaro, M., D'Asaro, M., Caccamo, N., Iovino, F., Francipane, M. G., Meraviglia, S., Orlando, V., La Mendola, C., Gulotta, G., Salerno, A., et al., 2009. Efficient killing of human colon cancer stem cells by $\gamma \delta$ t lymphocytes. The Journal of Immunology 182 (11), 7287-7296.

Vincent, T. L., Brown, J. S., 2005. Evolutionary game theory, natural selection, and Darwinian dynamics. Vol. 382. Cambridge University Press Cambridge.

Vives, M., Ginestà, M. M., Gracova, K., Graupera, M., Casanovas, O., Capellà, G., Serrano, T., Laquente, B., Viñals, F., 2013. Metronomic chemotherapy following the maximum tolerated dose is an effective antitumour therapy affecting angiogenesis, tumour dissemination and cancer stem cells. International Journal of Cancer 133 (10), 2464-2472.

Wakao, S., Kitada, M., Kuroda, Y., Shigemoto, T., Matsuse, D., Akashi, H., Tanimura, Y., Tsuchiyama, K., Kikuchi, T., Goda, M., et al., 2011. Multilineage-differentiating stress-enduring (muse) cells are a primary source of induced pluripotent stem cells in human fibroblasts. Proceedings of the National Academy of Sciences 108 (24), 9875-9880.

Wilensky, U., Rand, W., 2009. An introduction to agent-based modeling: Modeling natural, social and engineered complex systems with netlogo.

Yamashita, Y. M., Mahowald, A. P., Perlin, J. R., Fuller, M. T., 2007. Asymmetric inheritance of mother versus daughter centrosome in stem cell division. Science 315 (5811), 518-521.

Zipori, D., 2004. The nature of stem cells: state rather than entity. Nature Reviews Genetics 5 (11), 873-878. 


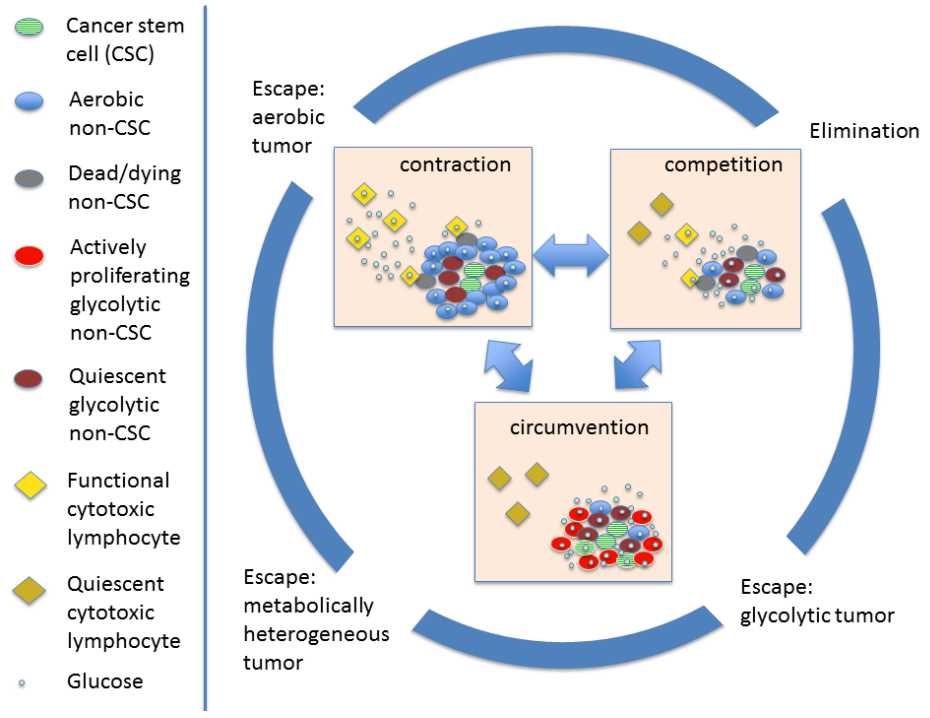

Figure 1: Schematic representation of the proposed immunoediting construct, which incorporates CSCs using aerobic and particularly glycolytic non-CSCs as a protective shield from cytotoxic lymphocytes.
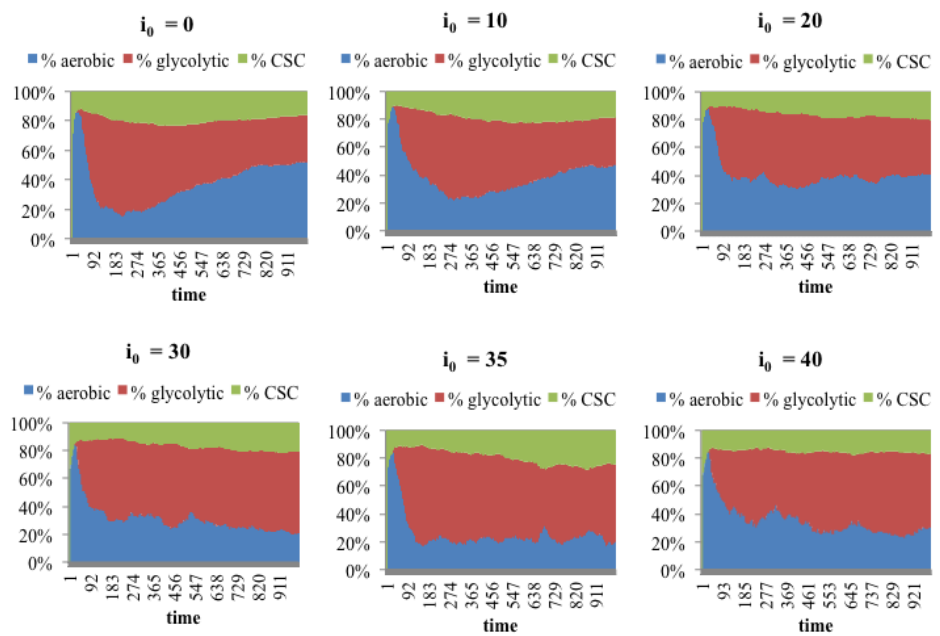

Figure 2: Change in tumor composition of CSCs (green) to glycolytic non-CSCs (red) to aerobic non-CSCs (blue) over time for varying initial states of the host immune system, represented by parameter $i_{0}$. As one can see, the proportion of glycolytic non-CSCs tends to increase with $i_{0}$ as CSCs require increased protection from CTLs. Threshold of sensitivity to oxygen for all cells is set to $\omega=5$. 


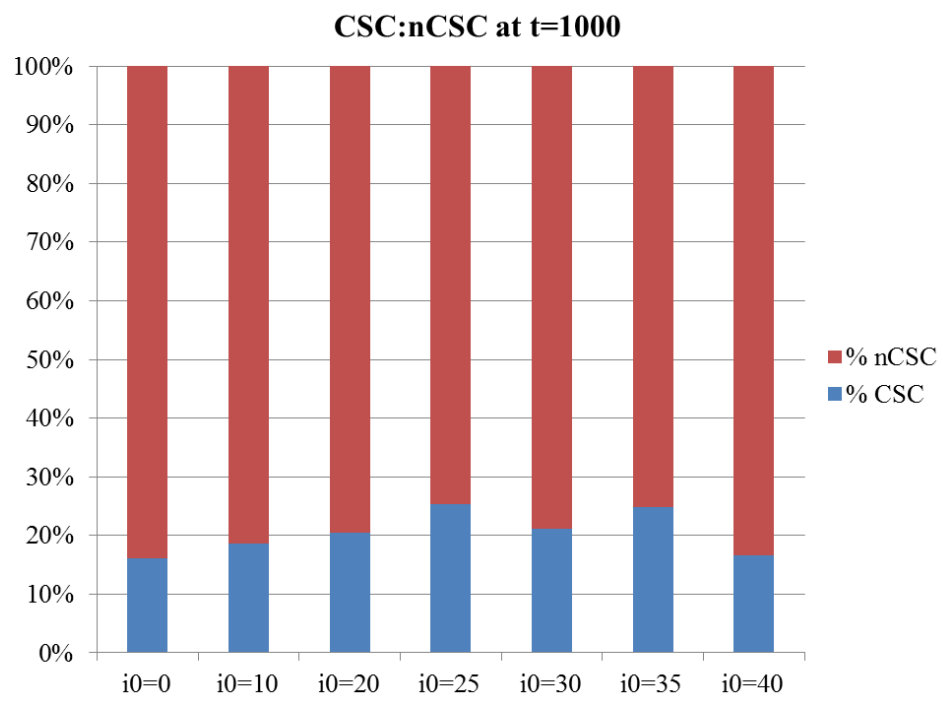

Figure 3: Proportion of CSCs and non-CSCs at $t=1000$ for all varying initial states of the host immune system, represented by parameter $i_{0}$. As one can see, the highest proportion of CSCs is observed not for immunodeficient or extremely immunocompetent host, but for intermediate values of $i_{0}$. Threshold of sensitivity to oxygen for all cells is set to $\omega=5$. 

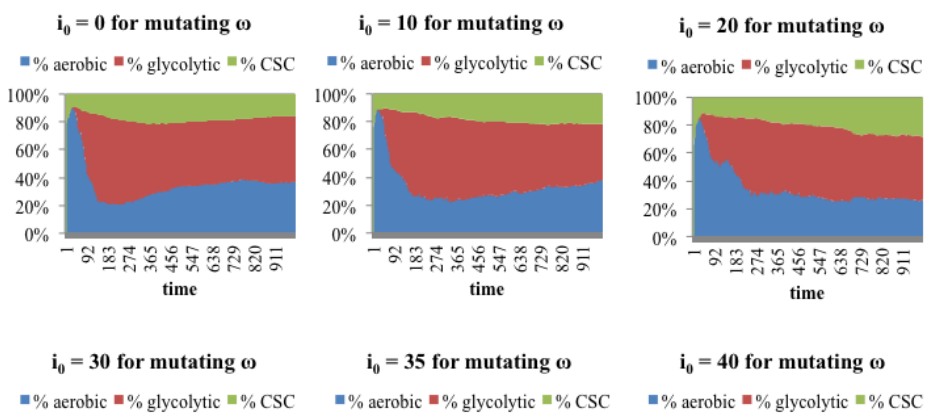

= $\%$ aerobic $=\%$ glycolytic $=\%$ CSC
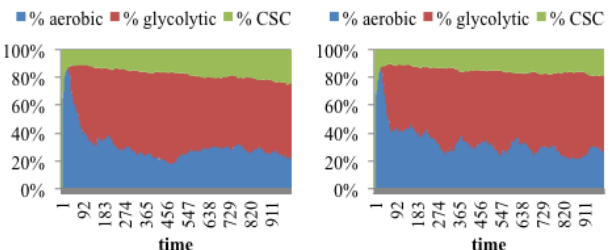

Figure 4: Change in tumor composition of CSCs (green) to glycolytic non-CSCs (red) to aerobic non-CSCs (blue) over time for varying initial states of the host immune system, represented by parameter $i_{0}$. As one can see, the proportion of glycolytic non-CSCs tends to increase with $i_{0}$ as CSCs require increased protection from CTLs. Threshold of sensitivity to oxygen for all cells is initiated at $\omega=5$ but is allowed to mutate with each cell division for each cell. 
CSC:nCSC at $\mathbf{t}=\mathbf{1 0 0 0}$ for mutating $\omega$

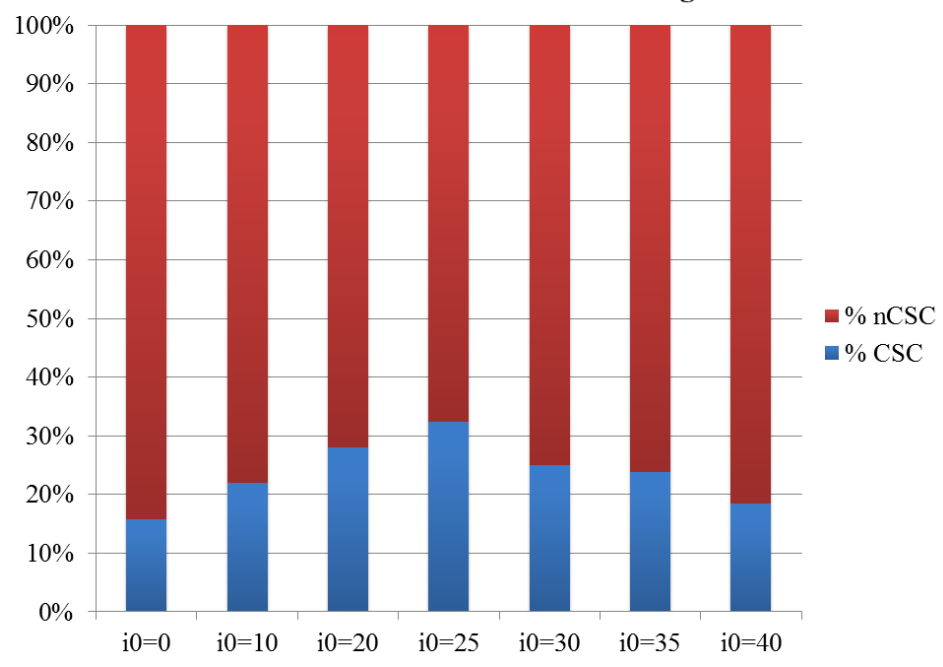

Figure 5: Proportion of CSCs and non-CSCs at $t=1000$ for all varying initial states of the host immune system, represented by parameter $i_{0}$. As one can see, the highest proportion of CSCs is observed not for immunodeficient or extremely immunocompetent host, but for intermediate values of $i_{0}$. Threshold of sensitivity to oxygen for all cells is initiated at $\omega=5$ but is allowed to mutate with each cell division for each cell. 

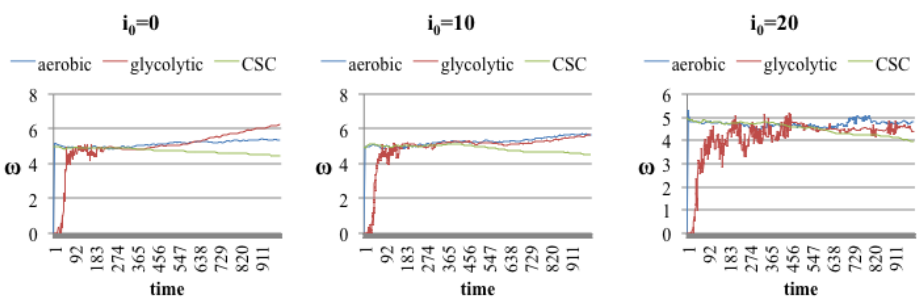

$\mathbf{i}_{0}=\mathbf{3 0}$

$\mathrm{i}_{0}=35$

time

- aerobic - glycolytic - CSC

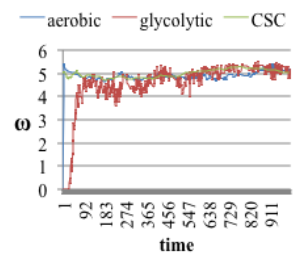

$\mathbf{i}_{0}=\mathbf{4 0}$

- aerobic - glycolytic - CSC

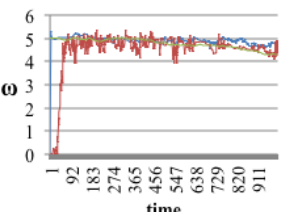

tim

time

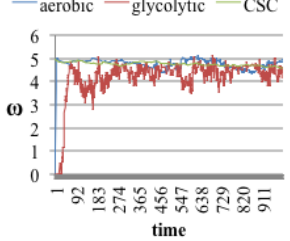

Figure 6: Change in the mutating threshold of sensitivity to oxygen (parameter $\omega=5$ ) over time for varying initial states of the host immune system, represented by parameter $i_{0}$. As one can see, while $\omega$ oscillates increasingly for larger $i_{0}$, the mean of $\omega=5$ remains largely the same for all three cell types. 


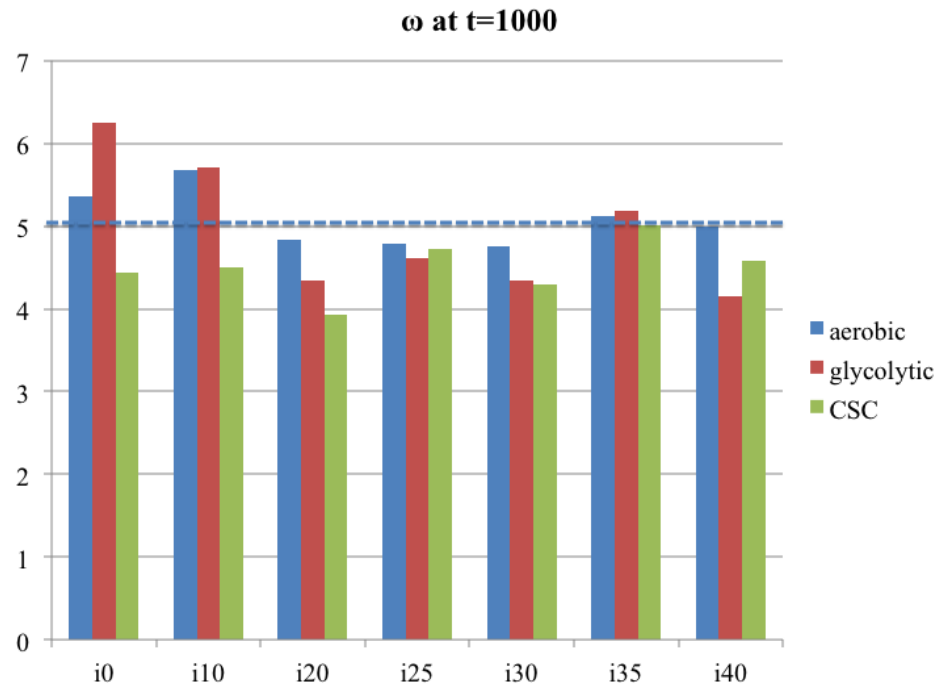

Figure 7: Mean value of the threshold of sensitivity to oxygen (parameter $\omega=5$ ) at $t=1000$ for varying initial states of the host immune system, represented by parameter $i_{0}$, compared to fixed value of $\omega=5$ for the first set of simulations. While there are slight changes in $\omega=5$ for all three cell types, none of them were statistically significant as evaluated by a 2 -tailed type 1 t-test, suggesting that it is not oxygen availability. 